Case Report

\title{
Molecular Monitoring in Adult Philadelphia Chromosome-Positive Acute Lymphoblastic Leukemia with the Variant e13a3 BCR-ABL1 Fusion
}

\author{
Mireille Crampe, ${ }^{1}$ Laura Kearney, ${ }^{1}$ David O'Brien ${ }^{D},{ }^{2}$ C. Larry Bacon, ${ }^{2}$ Derville O'Shea, ${ }^{3}$ \\ and Stephen E. Langabeer $\mathbb{D}^{1}$
}

${ }^{1}$ Cancer Molecular Diagnostics, St. James's Hospital, Dublin 8, Ireland

${ }^{2}$ Department of Haematology, St. James's Hospital, Dublin 8, Ireland

${ }^{3}$ Department of Haematology, Cork University Hospital, Cork, Ireland

Correspondence should be addressed to Stephen E. Langabeer; slangabeer@stjames.ie

Received 3 April 2019; Accepted 13 May 2019; Published 27 June 2019

Academic Editor: Akimichi Ohsaka

Copyright (c) 2019 Mireille Crampe et al. This is an open access article distributed under the Creative Commons Attribution License, which permits unrestricted use, distribution, and reproduction in any medium, provided the original work is properly cited.

\begin{abstract}
Monitoring $B C R-A B L 1$ transcript levels in patients with Philadelphia chromosome-positive acute lymphoblastic leukemia (Ph+ $\mathrm{ALL}$ ) is a widely adopted method to assess response to therapy. However, a small minority of Ph+ ALL patients express variant $B C R-A B L 1$ transcript types, usually due to splicing of alternative $B C R$ or $A B L 1$ exons. Whether patients expressing these rare, variant $B C R-A B L 1$ transcripts have a distinct phenotype or response to therapy is not known due to the limited number of reported cases. Here, we report the presenting features of Ph+ ALL in a young adult with a variant e13a3 BCR-ABL1 fusion. Molecular monitoring reflected the disease response from diagnosis through allogeneic stem cell transplantation which resulted in undetectable e13a3 $B C R-A B L 1$ transcripts. This case highlights the value of molecular monitoring in Ph+ ALL patients with variant $B C R-A B L 1$ transcripts and the requirement for standardization of such assays.
\end{abstract}

\section{Introduction}

Philadelphia chromosome-positive acute lymphoblastic leukemia $(\mathrm{Ph}+\mathrm{ALL})$ in adults is an aggressive disease that responds poorly to conventional chemotherapy. Despite improvements in survival with the addition of tyrosine kinase inhibitors (TKI) to chemotherapy, hematopoietic allogeneic stem cell transplantation (ASCT) remains the only curative option in those eligible patients [1]. Molecular monitoring of $B C R-A B L 1$ transcripts is a valuable tool in assessing individual patient response to chemotherapy and ASCT [2-4]. The most common BCR-ABL1 transcripts in $\mathrm{Ph}+\mathrm{ALL}$ are the e1a2, e13a2, and e14a2 fusions [5]; however, approximately $5 \%$ of adult patients express variant $B C R-A B L 1$ transcripts [6]. Of these variants, the e13a3 (b2a3) BCR-ABL1 type is extremely rare with scant information regarding optimal therapeutic approach $[7,8]$.
Characterisation of these rare $B C R-A B L 1$ variants also affords the selection of appropriate primer/probe combinations for reverse-transcriptase quantitative PCR (RT-qPCR) assessment of residual disease. The presentation and clinical course of a patient with e13a3 BCR-ABL1 Ph+ ALL is reported.

\section{Case Report}

A 23-year-old male presented with chest pain and dyspnoea, a hemoglobin count of $7.4 \mathrm{~g} / \mathrm{dL}$, platelet count of $<10 \times 10^{9} /$ $\mathrm{L}$, and white cell count of $11.6 \times 10^{9} / \mathrm{L}$. Bone marrow (BM) biopsy and aspirate demonstrated a $95 \%$ infiltration of lymphoblasts (Figure 1). Immunophenotyping of the BM aspirate showed lymphoblasts were CD10-, CD19-, CD20-, CD34-, TdT-, and HLA-DR-positive. Cytogenetic analysis revealed a complex clone in 10 cells analysed by G-banding 


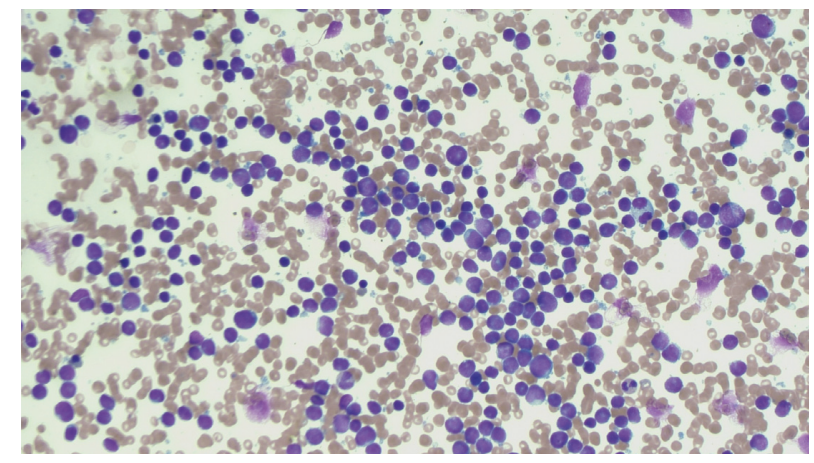

FIGURE 1: Bone marrow morphology at diagnosis showing infiltration by lymphoblasts.

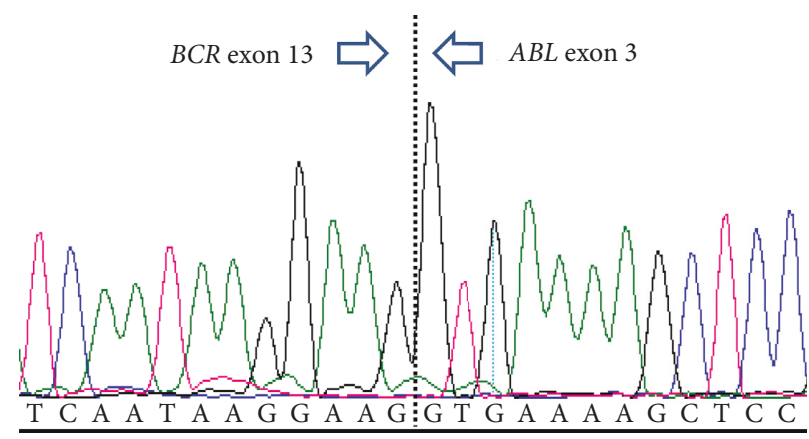

Figure 2: Sanger sequencing demonstrating presence of the e13a3 BCR-ABL1 fusion gene.

containing various structural and numerical abnormalities including a derivative chromosome 22 from a translocation between the long arms of chromosomes 9 and 22. The composite karyotype was 44-48, XY, $+X, t(1 ; 14)(\mathrm{p} 32 ; \mathrm{q} 32),-3$, $-6, \operatorname{add}(6)(\mathrm{p} 21), \operatorname{add}(7)(\mathrm{p} 21), \operatorname{add}(8)(\mathrm{p} 21),-10, \operatorname{add}(12)(\mathrm{p} 13)$, $+16, \operatorname{add}(19)(\mathrm{p} 13), \operatorname{der}(22) t(9 ; 22)(\mathrm{q} 34 ; \mathrm{q} 11.2),+1 \sim 2 \mathrm{mar}$, inc [cp10]. Interphase FISH analysis showed the presence of $B C R$ $A B L 1$ rearrangement in 64/100 cells analysed. Standardised RT-PCR and Sanger sequencing demonstrated e13a3 BCR$A B L 1$ transcripts [9] (Figure 2). E13a3 BCR-ABL1 transcripts lack $A B L 1$ exon a2, thus prohibiting the use of a standardised primer/probe combination for e13a2/e14a2 BCR-ABL1 qPCR [10]. A modified $B C R-A B L 1 \mathrm{qPCR}$ assay was therefore adopted utilising $B C R$ forward primer ENF501F2 [9] with $A B L 1$ reverse primer ENR1063 and $A B L 1$ probe ENP1043, the latter both complementary to $A B L 1$ exon a3 sequence [11]. The $B C R-$ $A B L 1$ standard curve was constructed using serial dilutions of an e14a3 $B C R-A B L 1$ plasmid over a five log range $(100 \%$ to $0.001 \% B C R-A B L 1$ transcripts) with $A B L 1$ as the reference gene as previously described [12]. Best practice guidelines for $B C R-A B L 1 \mathrm{qPCR}$ and data interpretation were followed [13]. Presentation e13a3 $B C R-A B L 1$ transcripts were of a high level (BCR-ABL1/ABL1 75.7\%) consistent with a diagnosis of prepre-B cell $\mathrm{Ph}+\mathrm{ALL}$.

The patient commenced induction treatment with rituximab, dexamethasone, vincristine, and daunorubicin, with imatinib $400 \mathrm{mg}$ oral daily started on day 15. After phase-one induction, the bone marrow aspirate demonstrated a complete morphological response with residual disease detected by immunophenotyping and RT-qPCR
(BCR-ABL1/ABL1 8.11\%). After phase-two induction, the bone marrow $B C R-A B L 1 / A B L 1$ level had fallen to $0.053 \%$. Following high-dose methotrexate, the pre-ASCT $B C R$ $A B L 1$ level was $0.034 \%$. The patient proceeded to ASCT from an unrelated donor after cyclophosphamide and total body irradiation conditioning and recommenced continued imatinib maintenance. $B C R-A B L 1$ transcripts were not detected in the peripheral blood at one, two, and three and a half months post-ASCT (Figure 3). Continued close molecular monitoring is planned.

\section{Discussion}

E13a3 $B C R-A B L 1$ transcripts lack $A B L 1$ exon a2 that encodes part of $\mathrm{SH} 3$ domain thought to contribute to leukemogenesis by inhibition of the kinase domain and by STAT 5 activation [6]. In chronic myeloid leukemia patients, this transcript results in an indolent and TKI-responsive form of disease [14-16]; however, its prognostic significance in adult $\mathrm{Ph}+\mathrm{ALL}$ patients remains unknown due to the limited number of annotated cases. While the possibility exists of lymphoid blast crisis in chronic myeloid leukemia (CML), this transformation in e13a3 $B C R-A B L 1 C M L$ is rare [17]. In the absence of basophilia, thrombocytosis, and splenomegaly, this case likely represents de novo Ph+ ALL. Additional chromosomal abnormalities and complex karyotypes are frequently observed in $\mathrm{Ph}+\mathrm{ALL}$, as witnessed in this case. There is some suggestion that in Ph+ ALL, additional cytogenetic abnormalities are associated with a shorter overall survival and might therefore be used for 


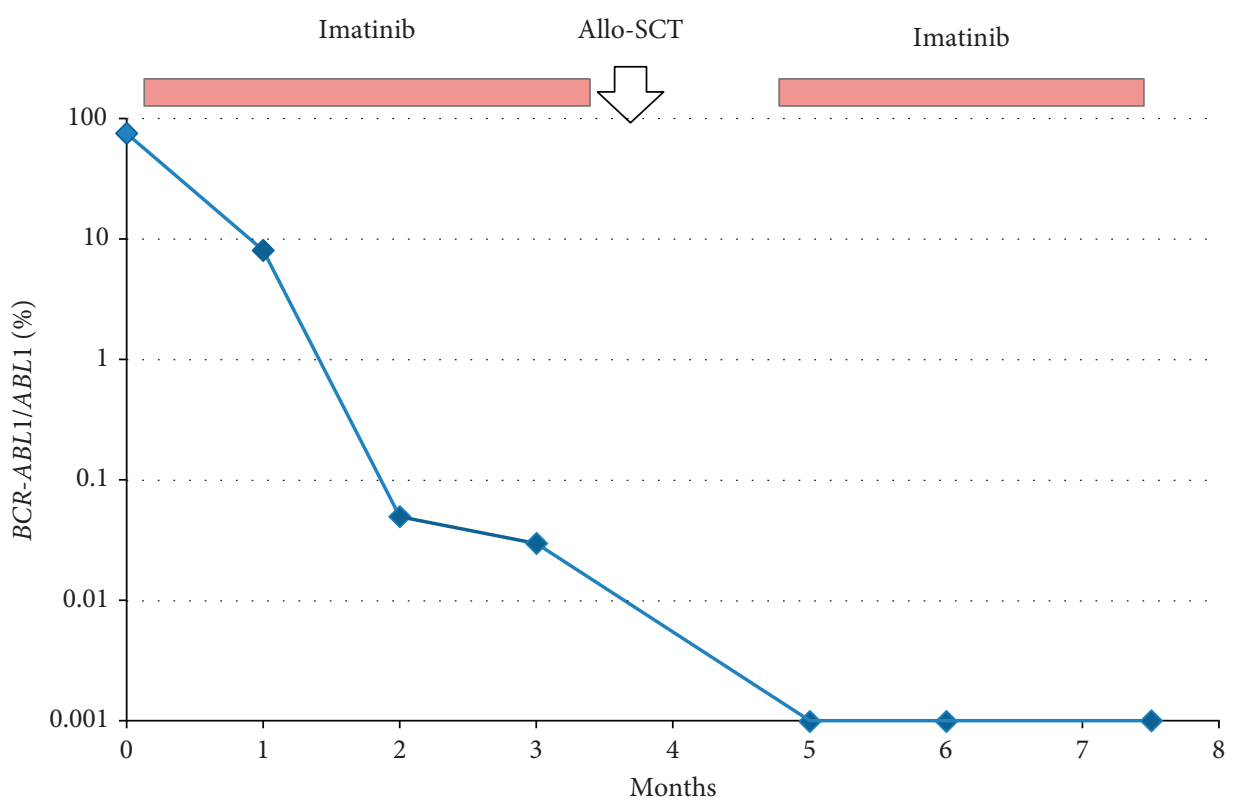

FIgURE 3: Molecular monitoring of e13a3 BCR-ABL1 transcripts throughout clinical course. AlloSCT: allogeneic stem cell transplantation.

stratification purposes [18]. In the post-ASCT setting, monitoring $B C R-A B L 1$ transcript levels is an essential component of $\mathrm{Ph}+\mathrm{ALL}$ patient management [19] with effective standardisation of RT-qPCR assays for this and other variant $B C R-A B L 1$ fusion transcripts required [20].

Reporting of further cases would enable identification of any phenotypic characteristics of e13a3 BCR-ABL1 Ph+ ALL and help establishing optimal treatment strategies for patients with this rare genotype.

\section{Conflicts of Interest}

The authors declare that there are no conflicts of interest regarding the publication of this paper.

\section{References}

[1] M. Yilmaz, H. Kantarjian, F. Ravandi-Kashani, N. J. Short, and E. Jabbour, "Philadelphia chromosome-positive acute lymphoblastic leukemia in adults: current treatments and future perspectives," Clinical Advances in Hematology \& Oncology, vol. 16, no. 3, pp. 216-223, 2018.

[2] M. Yanada, I. Sugiura, J. Takeuchi et al., "Prospective monitoring of BCR-ABL1 transcript levels in patients with Philadelphia chromosome-positive acute lymphoblastic leukaemia undergoing imatinib-combined chemotherapy," British Journal of Haematology, vol. 143, no. 4, pp. 503-510, 2008.

[3] L. Zhang, R. T. Ramjit, C. E. Hill, M. Arellano, H. J. Khoury, and K. P. Mann, "Clinical significance of quantitative monitoring and mutational analysis of BCR-ABL1 transcript in Philadelphia chromosome positive B lymphoblastic leukemia," Leukemia \& Lymphoma, vol. 57, no. 2, pp. 364-369, 2016.

[4] G. Cazzaniga, P. De Lorenzo, J. Alten et al., "Predictive value of minimal residual disease in Philadelphia-chromosomepositive acute lymphoblastic leukemia treated with imatinib in the European intergroup study of post-induction treatment of Philadelphia-chromosome-positive acute lymphoblastic leukemia, based on immunoglobulin/T-cell receptor and BCR/ABL1 methodologies," Haematologica, vol. 103, no. 1, pp. 107-115, 2018.

[5] S. E. Langabeer, "Variant BCR-ABL1 fusion genes in adult Philadelphia chromosome-positive B-cell acute lymphoblastic leukemia," EXCLI Journal, vol. 16, pp. 1144-1147, 2017.

[6] A. Tuszynski, S. Dhut, B. D. Young et al., "Detection and significance of bcr-abl mRNA transcripts and fusion proteins in Philadelphia-positive adult acute lymphoblastic leukemia," Leukemia, vol. 7, no. 10, pp. 1504-1508, 1993.

[7] T. Burmeister, S. Schwartz, A. Taubald et al., "Atypical BCR-ABL mRNA transcripts in adult acute lymphoblastic leukemia," Haematologica, vol. 92, no. 12, pp. 1699-1702, 2007.

[8] X. Zhang and J. Pan, "An e13a3 BCR-ABL1 fusion transcript in variant $\mathrm{t}(9 ; 22 ; 17)(\mathrm{q} 34 ; \mathrm{q} 11 ; \mathrm{q} 21)$-positive adult acute lymphoblastic leukemia," International Journal of Laboratory Hematology, vol. 38, no. 3, pp. e52-e55, 2016.

[9] J. J. M van Dongen, E. A. Macintyre, J. A. Gabert et al., "Standardized RT-PCR analysis of fusion gene transcripts from chromosome aberrations in acute leukemia for detection of minimal residual disease," Leukemia, vol. 13, no. 12, pp. 1901-1928, 1999.

[10] J. Gabert, E. Beillard, V. H. J. van der Velden et al., "Standardization and quality control studies of 'real-time' quantitative reverse transcriptase polymerase chain reaction of fusion gene transcripts for residual disease detection in leukemia-a Europe against cancer program," Leukemia, vol. 17, no. 12, pp. 2318-2357, 2003.

[11] E. Beillard, N. Pallisgaard, V. H. J. van der Velden et al., "Evaluation of candidate control genes for diagnosis and residual disease detection in leukemic patients using 'realtime' quantitative reverse-transcriptase polymerase chain reaction (RQ-PCR)-a Europe against cancer program," Leukemia, vol. 17, no. 12, pp. 2474-2486, 2003.

[12] S. L. McCarron, S. E. Langabeer, K. Bolger et al., "Molecular response to imatinib in chronic myeloid leukemia with a variant e13a3 BCR-ABL1 fusion," Medical Oncology, vol. 32, no. 2, p. 452, 2015. 
[13] L. Foroni, G. Wilson, G. Gerrard et al., "Guidelines for the measurement of BCR-ABL1 transcripts in chronic myeloid leukaemia," British Journal of Haematology, vol. 153, no. 2, pp. 179-190, 2011.

[14] D. S. Snyder, R. McMahon, S. R. Cohen, and M. L. Slovak, "Chronic myeloid leukemia with an e13a3 BCR-ABL fusion: benign course responsive to imatinib with an RT-PCR advisory," American Journal of Hematology, vol. 75, no. 2, pp. 92-95, 2004.

[15] B. Pienkowska-Grela, R. Woroniecka, I. Solarska et al., "Complete cytogenetic and molecular response after imatinib treatment for chronic myeloid leukemia in a patient with atypical karyotype and BCR-ABL b2a3 transcript," Cancer Genetics and Cytogenetics, vol. 174, no. 2, pp. 111-115, 2007.

[16] B. Liu, W. Zhang, and H. Ma, "Complete cytogenetic response to Nilotinib in a chronic myeloid leukemia case with a rare e13a3(b2a3) BCR-ABL fusion transcript: a case report," Molecular Medicine Reports, vol. 13, no. 3, pp. 2635-2638, 2016.

[17] J. Ha, J.-W. Cheong, S. Shin, S.-T. Lee, and J. R. Choi, "Chronic myeloid leukemia with rare variant b2a3 (e13a3) BCR-ABL1 fusion," Annals of Laboratory Medicine, vol. 36, no. 3, pp. 287-289, 2016.

[18] C. A. Seol, Y.-U. Cho, S. Jang et al., "Prognostic significance of recurrent additional chromosomal abnormalities in adult patients with Philadelphia chromosome-positive acute lymphoblastic leukemia," Cancer Genetics, vol. 216-217, pp. 2936, 2017.

[19] H. Pfeifer, B. Wassmann, W. Bethge et al., "Randomized comparison of prophylactic and minimal residual diseasetriggered imatinib after allogeneic stem cell transplantation for BCR-ABL1-positive acute lymphoblastic leukemia," Leukemia, vol. 27, no. 6, pp. 1254-1262, 2013.

[20] S. E. Langabeer, "Standardized molecular monitoring for VariantBCR-ABL1Transcripts in chronic myeloid leukemia," Archives of Pathology \& Laboratory Medicine, vol. 139, no. 8, p. 969, 2015. 


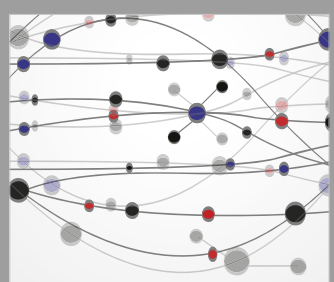

The Scientific World Journal
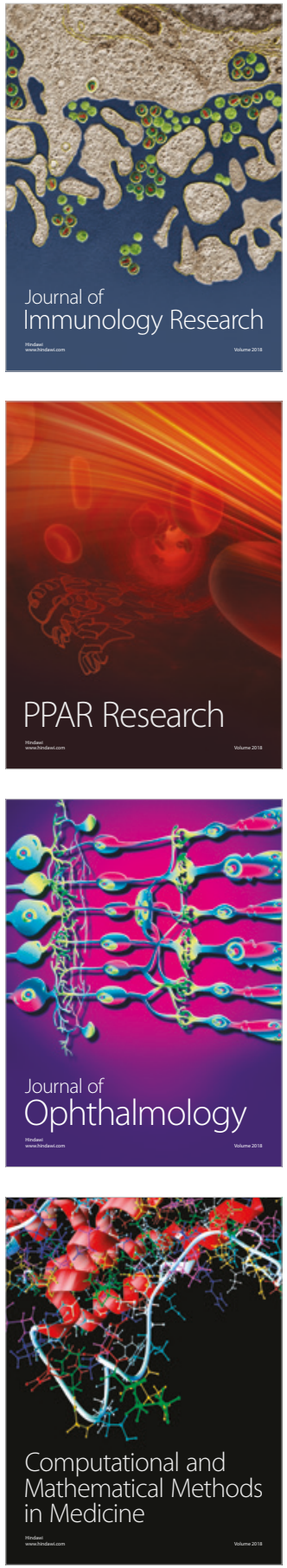

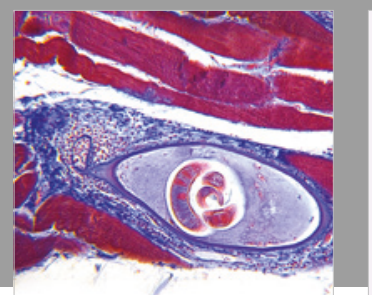

Gastroenterology Research and Practice

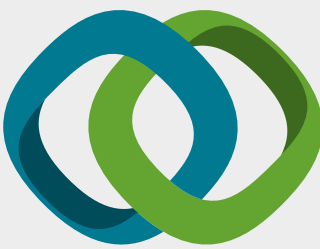

\section{Hindawi}

Submit your manuscripts at

www.hindawi.com
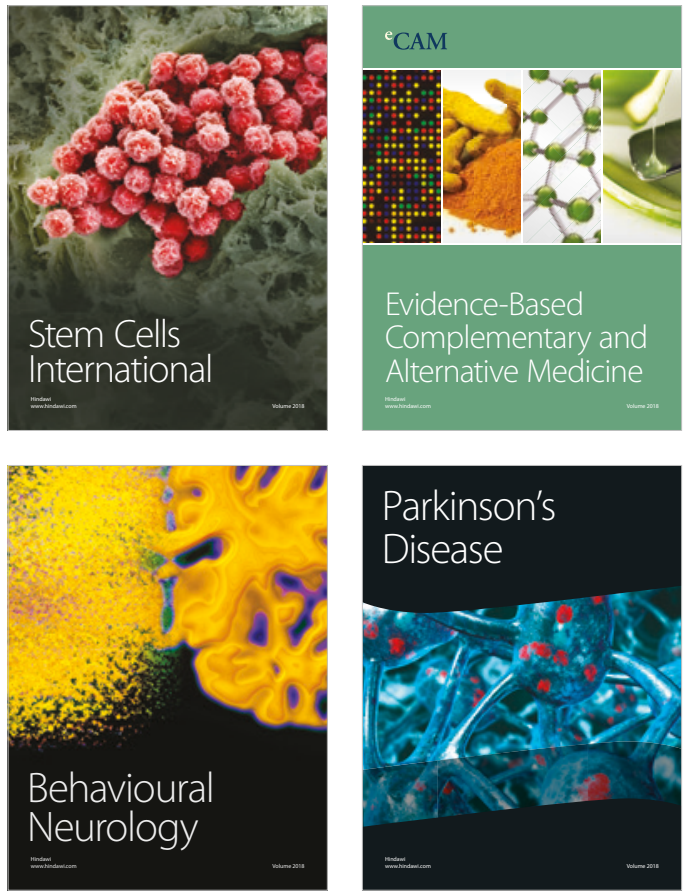

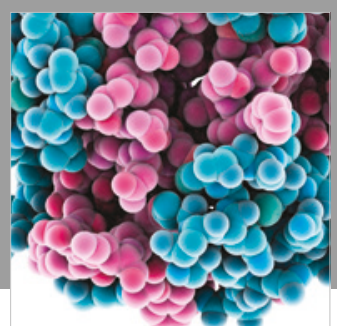

ournal of

Diabetes Research

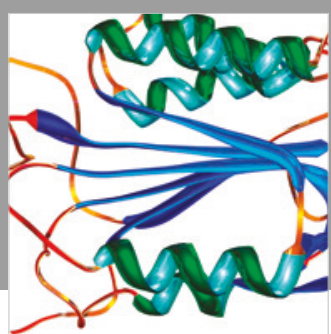

Disease Markers
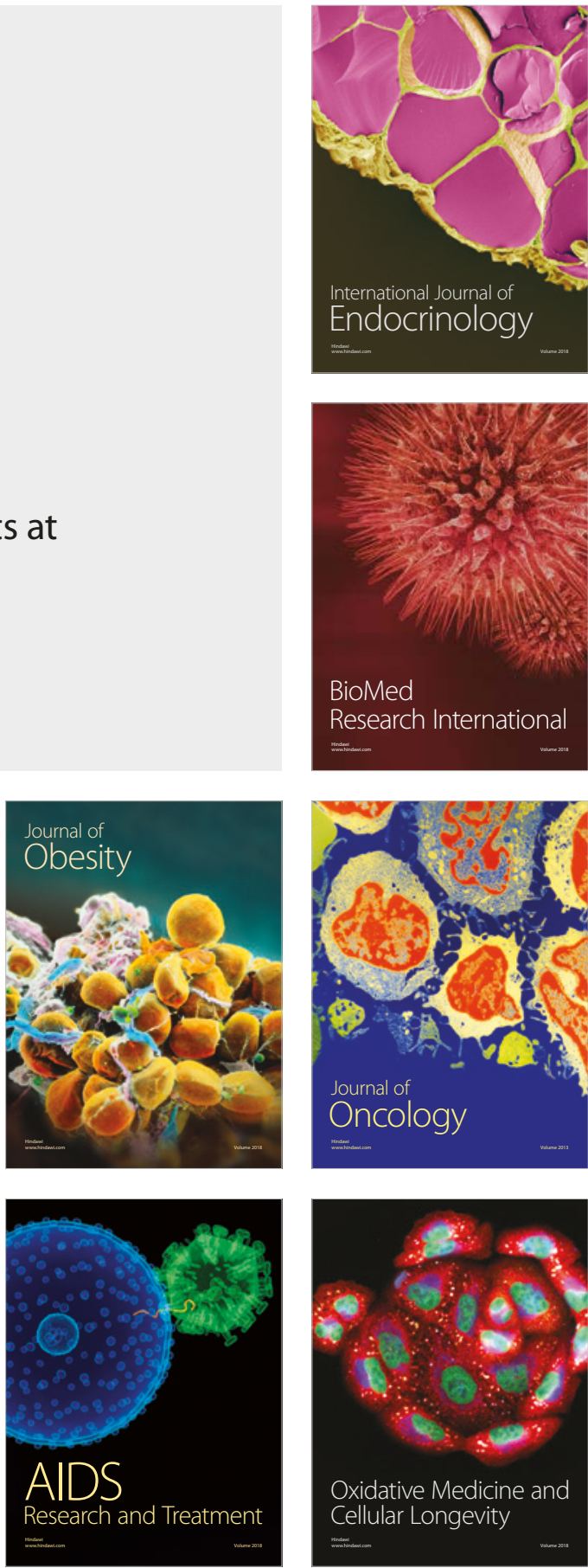